\title{
Stress Analysis of New Type Pre-Stressed Anchor Bearing Plate Combining Stamping with Welding Forming and Its Anchorage Zone
}

\author{
Daosen Chen ${ }^{1}$, Nianchun Deng ${ }^{2}$, Zanzhi Wang1, Haining Zuo ${ }^{3}$ \\ ${ }^{1}$ Department of Civil Engineering and Architecture, Guangxi University of Science and Technology, Liuzhou, China \\ ${ }^{2}$ Department of Civil Engineering and Architecture, Guangxi University, Nanning, China \\ ${ }^{3}$ Liuzhou OVM Machinery Co., Ltd., Liuzhou, China \\ Email: ^dengnch@163.com
}

How to cite this paper: Chen, D.S., Deng, N.C., Wang, Z.Z. and Zuo, H.N. (2017) Stress Analysis of New Type Pre-Stressed Anchor Bearing Plate Combining Stamping with Welding Forming and Its Anchorage Zone. World Journal of Engineering and Technology, 5, 33-41.

https://doi.org/10.4236/wjet.2017.54B004

Received: June 13, 2017

Accepted: October 9, 2017

Published: October 12, 2017

\begin{abstract}
An anchor bearing plate transfers the anchoring force from anchor plate to the concrete and the pre-stress is formed in the concrete structure. Currently, the main type of anchor bearing plate is cast iron. It is brittle during transportation and tension process. This paper presents a new type of anchor bearing plate combined stamping with welding forming. The structure of the new type anchor bearing plate is introduced. The stress states of the anchor bearing plate and anchorage zone under work are studied. Various specifications of anchor bearing plate are studied by ANSYS finite element analysis software following the AASHTO specification. The analysis results are compared with the results of the same type of OVM round-shaped anchor plate. The study results show that the new pre-stressed anchor plates combined stamping with welding forming are feasible and more sturdy which can meet the engineering demand.
\end{abstract}

\section{Keywords}

Pre-Stressed Concrete Structure, Finite Element Analysis,

Anchor Bearing Plate, Stress of Anchorage Zone

\section{Introduction}

The prestressing technology in our country has mushroomed in the past 20 years. Prestressed concrete structure due to its reasonable force, handsome ap- 
pearance, materials saving and other characteristics helps the development of modern engineering. The post-tensioned prestressed concrete structure is hold the prestressing tendons, which is transmitted the pre-stressed passed though the anchor plate and anchor plate, finally transmitted to concrete formed the prestressing force. We can find that the stress in the concrete area of the lower end of the anchor bearing plate is extremely complicated in the analysis of the anchor bearing plate. The compressive strength of concrete is increased, which is owe to the compressive deformation of the prestressing force the anchor bearing plate transmit and the lateral expansion of the concrete.

The component used to withstand the prestressing force the anchorage transmit and passed to the anchorage zone concrete is called the anchor bearing plate, where the prestressing anchorage is to be directly transferred to the concrete plate known as the common plate while the end plate and horn tube casting as the anchor bearing plate called casting pad plate [1]. According to statistics, China's annual needs a huge number of anchor bearing plate is about 20 million pieces, but the vast majority of anchor bearing plate just are put in production without a reasonable load transferring experiment which may be causing the construction of the anchor bearing plate cracking or concrete end area broken, thus an invaluable project loss.

\section{Design of New Type Anchor Bearing Plate for YM15-7D (HJ)}

\subsection{Present Situation and Defects of Casting Anchor Bearing Plate}

In the post-tensioned prestressed concrete members, the quality of the prestressed concrete is also improved due to the continuous optimization and improvement of the components such as the anchor bearing plate. If the pre-stressed transmitted to concrete from the anchor bearing plate is not uniform, it is easy causing longitudinal cracks or serious damage which is due to concentrate the concrete with a large stress. The structure of the anchor bearing plate is one of the main factors affecting the local pressure of the concrete. The reasonable structure can solve the stress concentration, deformation and cracking of the concrete.

The vast majority of the existing anchor bearing plate cast iron casting processing, the use of this process still exist shortcomings:

The anchor bearing plate prestressed strength is insufficient and easy damage which is due to used the recovery of miscellaneous material casting forming anchor bearing plate structure more loose. The anchor bearing plate is the forced fulcrum when the strand is tensioned and once damaged, it will cause an immeasurable security risks to the project.

Manufacturing methods of casting anchor bearing plate are high energy consumption, easy to pollute the environment, and due to its complex structure, it cannot be continuous production, so the production efficiency is low and the cost is high. 


\subsection{Presents YM15-7D (HJ) New Type Anchor Bearing Plate and the Design Idea}

This paper presents the YM15-7D (HJ) new type seven-hole round tower-shaped anchor bearing plate (Figure 1), its new intention lies in the formation of steel plate combined stamping with welding. Its material is more compact than the traditional anchor bearing plate and it can withstand a higher stress. Even when it is subjected to yielding pressure, only deformation occurs without crushing and it can still play a supporting role. Stamping process can achieve continuous production or even automated production, significantly improve production efficiency and reduce production costs.

The stress concentration area of the anchor bearing plate is mainly distributed in the bottom of the pressure bearing surface. Therefore, when designing the new type anchor bearing plate of YM15-7D (HJ), the multi-level step structure is arranged to the bottom of the pressure surface, which not only solves the unfavorable factors brought about by the stress concentration of the impact, but also makes full use of the multi-level step of the good transmission performance and saving the material. Reasonable increase in the anchor bearing plate and concrete pressure projection area are conducive to the uniform transmission of the load and the anchor bearing plate and concrete work together with the higher applicability [2]. The steps in the bearing load distribution and transmission process have the following functions [3]: 1) Reinforcement of the stiffness of the anchor bearing plate itself; 2) The transmission load and related to the location of the transmission area; 3) The deformation coordination ensures the prestress structure of the security.
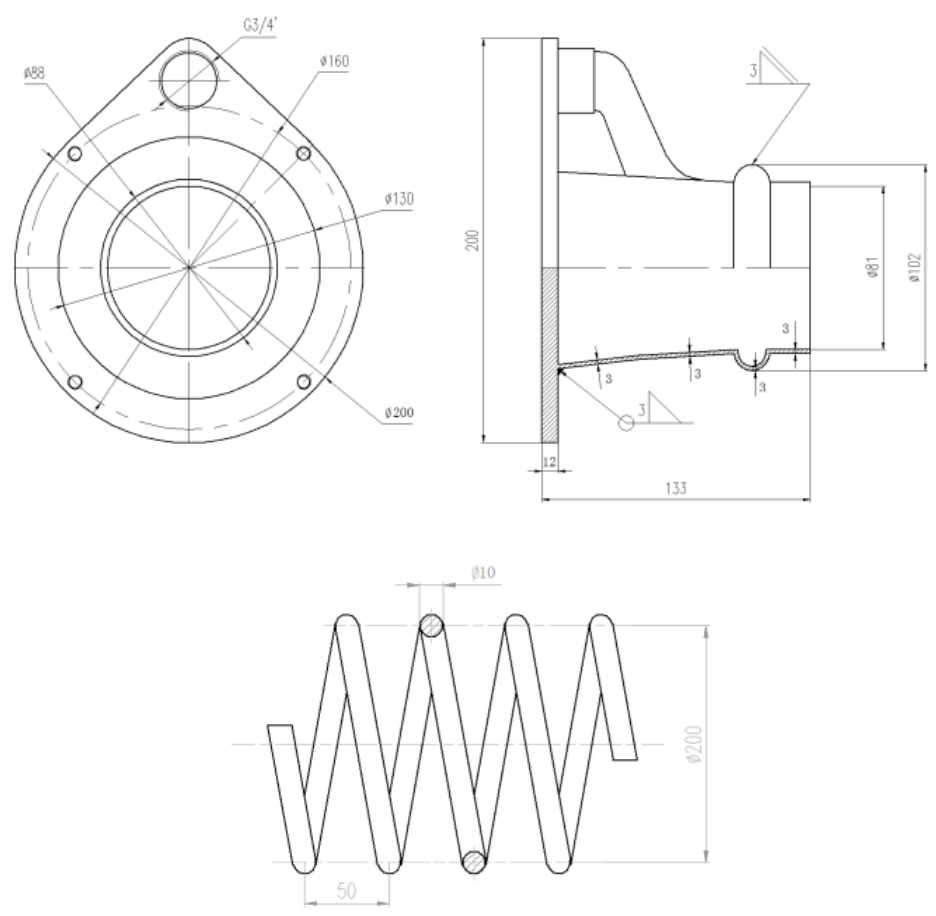

Figure 1. YM15-7D (HJ) new anchor bearing plate and spiral bar diagram. 


\subsection{Checking Calculation of the Concrete Bearing Capacity of YM15-7D (HJ) New Type Anchor Plate Anchor}

The load bore by the concrete structure will directly affect the effect of prestressing in the prestressed anchorage system [4], therefore, the security for the concrete structure should be checked. This article will check it with the related requirements in the specification of "AASHTO LRFD Bridge Design Specifications" [5]. The comparative analysis M15E-7D (TB) round tower-shaped anchor bearing plate has been checked and put into use, so no checking is here.

YM15-7D (HJ) new type anchor bearing plate as shown in Figure 1, the first step and the last step are the main bearing body. The design of the first step and the last step distance is large enough to superimpose the extreme value of concrete tensile stress, the anchor bearing plate of the effective bearing area can be seen as multiple bearing area overlay in the theoretical calculation.

According to the specification, the calculated compressive stress $f_{c a}$ of the anchorage zone concrete should not exceed the specified limit, i.e.:

$$
f_{c a}=\frac{0.6 P_{u} \kappa}{A_{b}\left(1+\ell_{c}\left(\frac{1}{b_{\text {eff }}}-\frac{1}{t}\right)\right)} \leq 0.7 \varphi f_{c i}^{\prime}
$$

where:

$P_{u}$-factored tendon force (N). According to 7 hole tension control force $P_{u}=1367100 \mathrm{~N} ;$

$\kappa$-correction factor for closely spaced anchorages. In this component, $\kappa=1$

$A_{b}$-effective bearing area $\left(\mathrm{mm}^{2}\right)$. In this component,

$$
A_{b}=A_{b 1}+A_{b 2}=\left[\left(200^{2}-89^{2}\right)+\left(104^{2}-88^{2}\right)\right] \times \pi / 4=27593.54 \mathrm{~mm}^{2} ;
$$

$b_{\text {eff }}$-lateral dimension of the effective bearing area measured parallel to the larger dimension of the cross-section $(\mathrm{mm})$. In this component, $b_{\text {eff }}=200 \mathrm{~mm}$;

$\ell_{c}$-longitudinal extent of confining reinforcement of the local zone but not more than the larger of $1.15 b_{\text {eff }}(\mathrm{mm})$. In this component, $\ell_{c}=50 \times 4+12=212 \mathrm{~mm}, \quad \ell_{c} \leq 1.15 b_{\text {eff }}$;

$t$-member thickness (mm). In this component, $t=360(\mathrm{~mm})$;

$\varphi$-Pressure coefficient. In this component, $\varphi=1$;

$f_{c i}^{\prime}$-Standard Strength of Concrete at Tension (MPa). This component uses C50 concrete, so

$$
\begin{gathered}
f_{c a}=\frac{0.6 P_{u} \kappa}{A_{b}\left(1+\ell_{c}\left(\frac{1}{b_{\text {eff }}}-\frac{1}{t}\right)\right)}=\frac{0.6 \times 1367100 \times 1}{27593.54 \times\left[1+212\left(\frac{1}{200}-\frac{1}{360}\right)\right]}=20.21 \mathrm{MPa} \\
0.7 \varphi f_{c i}^{\prime}=0.7 \times 1 \times 32.4=22.68 \mathrm{MPa}
\end{gathered}
$$




$$
f_{c a}=\frac{0.6 P_{u} \kappa}{A_{b}\left(1+\ell_{c}\left(\frac{1}{b_{e f f}}-\frac{1}{t}\right)\right)} \leq 0.7 \varphi f_{c i}^{\prime}
$$

Therefore, the formula is meet the specification design requirements.

\section{ANSYS Overall Model Analysis}

\subsection{Model Overview}

The finite element method has also been rapid development and gradually applied to the field of civil engineering along with the extensive application of computers, can accurately analyze the internal force of complex structures [6]. The ANSYS finite element analysis should be conducted to ensure the rationality of the design of the new type structure anchor bearing plate. As we known, the non-linear analysis of the material is the most common. In this paper, established the finite element model is based on the actual situation. Using C50 concrete and the size dimension to $360 \mathrm{~mm} \times 360 \mathrm{~mm} \times 720 \mathrm{~mm}$, which adopts the solid 65 unit of 8 nodes to divide the hexahedral mesh. The spiral stirrups selected Q235Ф10 mm with a total of 4 laps and adopts the pipe 16 unit to divide the grid. The anchor bearing plate selected Q235 is the ideal elastoplastic material which divided into hexahedral mesh by solid 186 unit.

Under the premise of not affecting the overall force of the model, we should calculate the analysis by the symmetry of the structure of the selected model $1 / 4$ (Figure 2) to reduce the number of computing units and speed up the calculation of convergence speed. Assuming that the material is continuous and uniform, the material type and parameters are shown in Table 1.

\subsection{Model Processing}

Putting the prestressing force to the anchor bearing plate and the concrete could increase of the contact area, the contact pressure and the friction force. It can be

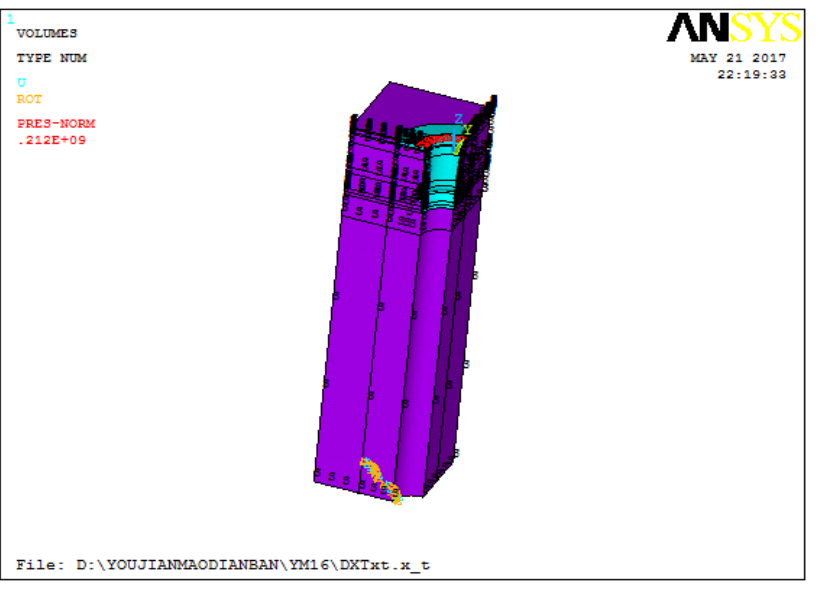

(a)

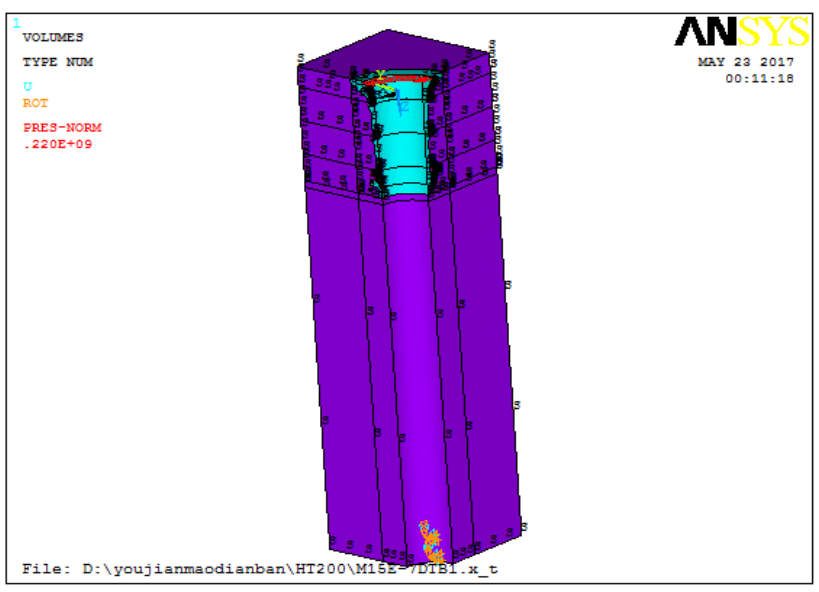

(b)

Figure 2. 1/4 model processing diagram of (a) YM15-7D (HJ) anchor bearing plate and (b) M15E-7D (TB) anchor bearing plate. 
Table 1. Material type and parameter table.

\begin{tabular}{ccccc}
\hline Material type & $\begin{array}{c}\text { Elastic } \\
\text { modulus } \\
(\mathrm{GPa})\end{array}$ & $\begin{array}{c}\text { Poisson's } \\
\text { ratio }\end{array}$ & $\begin{array}{c}\text { Yield } \\
\text { strength } \\
(\mathrm{MPa})\end{array}$ & $\begin{array}{c}\text { Quality } \\
(\mathrm{kg})\end{array}$ \\
\hline C50 concrete & 34.5 & 0.2 & $/$ & $/$ \\
$\begin{array}{c}\text { Q235 } \\
\text { (anchor bearing plate })\end{array}$ & 206 & 0.25 & 235 & 3 \\
$\begin{array}{c}\text { HT200 cast iron } \\
\text { (anchor bearing plate })\end{array}$ & 120 & 0.3 & $/$ & 4.74 \\
$\begin{array}{c}\text { Longitudinal } \\
\text { reinforcement, } \\
\text { stirrups and spiral bars }\end{array}$ & 210 & 0.3 & $/$ & / \\
\hline
\end{tabular}

determined that the anchor bearing plate and the concrete restrict each other deformation of the contact surface. Therefore, it is necessary to make contact analysis between the anchor bearing plate and the concrete contact surface in the model.

The most common way to solve the problem of contact is to contact the iterative method, which is based on the direct constraint of the contact algorithm. For contact problems, ANSYS processor comes with two kinds of contact analysis methods, one for the "rigid body-flexible body" contact, and the other for the "soft body-flexible body" contact. "Flexible-flexible body" contact is used in this paper and one of the boundary surfaces is called "target surface", the other boundary surface is called "contact surface". This model selects the contact surface of the anchor bearing plate as the "target surface", the concrete contact surface as the "contact surface", and the appropriate parameter setting to complete the contact treatment in the ANSYS. In this paper, we set in the contact surface and added the friction coefficient of 0.6 between in the anchor bearing plate with the concrete.

Mesh partitioning is one of the key problems in finite element analysis. According to the shape of the initial model, combining with Boolean operation and a variety of meshing methods such as sweep, mapped and free are used to get the best finite element model. As shown in Figure 3, YM15-7D (HJ) and M15E-7D (TB) are the ANSYS finite element models divided by sweeping strategy, in which the YM15-7D (HJ) model is divided into 7874 units, and the M15E-7D (TB) model is divided into 8212 units.

YM15-7D (HJ) new type anchor bearing plate and M15E-7D (TB) casting anchor bearing plate are all seven-hole round tower-shaped, the actual load of $260,400 \times 7 \times 0.75=1,367,100 \mathrm{~N}$, with uniform load the form acts on the end area of the inner ring of the anchor bearing plate. Add symmetric constraints to the two cutting faces of the model, and adds a three-way full constraint at the bottom, as shown in Figure 2. 


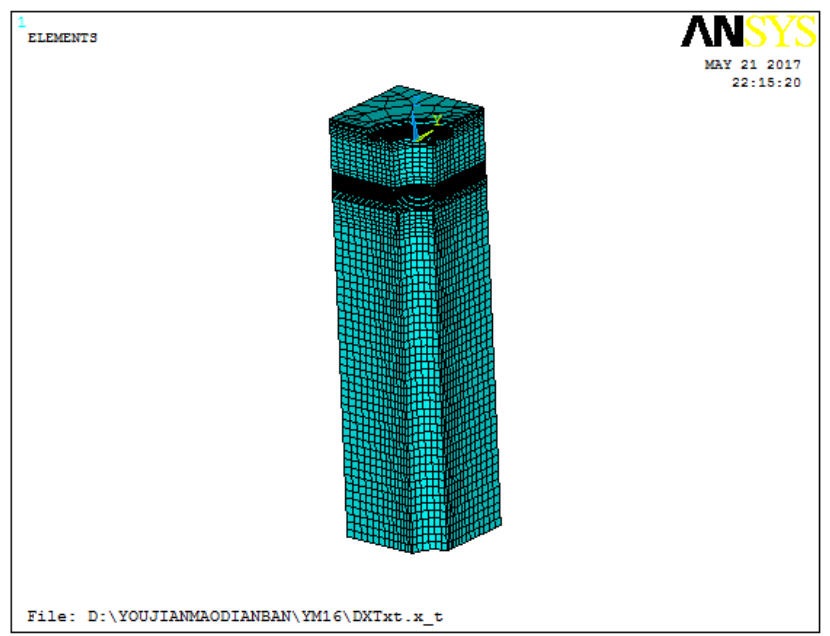

(a)

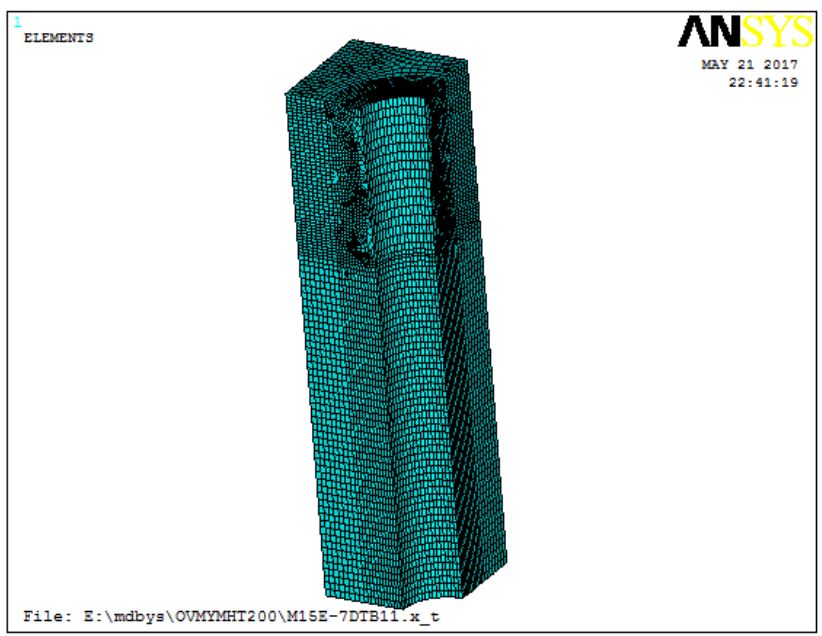

(b)

Figure 3. 1/4 model grid diagram of (a) YM15-7D (HJ) anchor bearing plate and (b) M15E-7D (TB) anchor bearing plate.

\section{The Results of Analysis}

Analyses were conducted on the YM15-7D (HJ) and the M15E-7D (TB) model according to the ANSYS finite element software. The results are as follows:

1) Observed YM15-7D (HJ) and M15E-7D (TB) anchor bearing plate stress cloud diagram shown in Figure 4, we can see the anchor bearing plate red part is the stress concentration area; the yellow section is the yield area. The maximum stress concentration area of the YM15-7D (HJ) anchor bearing plate does not affect the use of the anchor bearing plate. The yield area of the YM15-7D (HJ) anchor bearing plate mainly occurs at the step surface in contact with the concrete. The stress value is about $137 \mathrm{MPa}$ is less than the tensile strength of 235 MPa material. M15E-7D (TB) anchor bearing plate to remove the stress concentration section, the yield area stress value of about $106 \mathrm{MPa}$ is less than the tensile strength of $200 \mathrm{MPa}$ material. It can be seen that the stress of the two kinds of material anchor bearing plate is not much difference and all meet the standard design requirements.

2) Figure 5 shows the stress cloud of two kinds of spiral tendons in which we can see that the stress concentration area of the spiral tendons of YM15-7D $(\mathrm{HJ})$ model is larger and the maximum concentrated stress value is about 49.2 MPa. But the stress concentration area of the spiral tendons of the M15E-7D (TB) model is small; the yield stress value is about $157 \mathrm{MPa}$, all less than the tensile strength of $235 \mathrm{MPa}$ material, which meets the requirements of the specification.

3) Figure 6 shows the stress cloud of concrete under the anchors of YM15-7D (HJ) and M15E-7D (TB) model. In this paper, selection the calculated results of the ultimate tensile stress values of C50 concrete is among $-5 \mathrm{MPa}$ with $5 \mathrm{MPa}$. It can be seen from Figure 6 that the stress values of the two models are within 5 $\mathrm{MPa}$, which are less than the tensile and compressive strength of C50 concrete, which meet the requirements of the specification. The YM15-7D (HJ) model stress is more uniform than the M15E-7D (TB) model. 


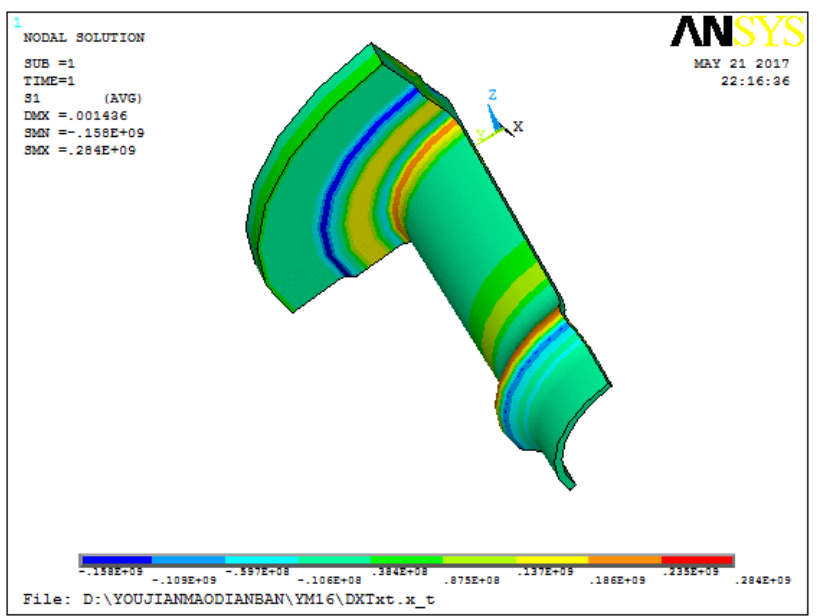

(a)

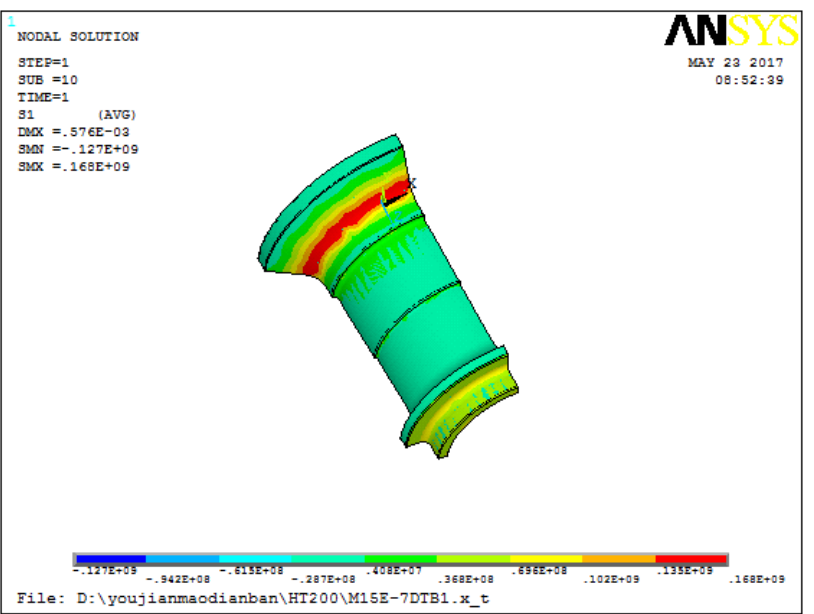

(b)

Figure 4. Stress cloud of (a) YM15-7D (HJ) anchor bearing plate and (b) M15E-7D (TB) anchor bearing plate.

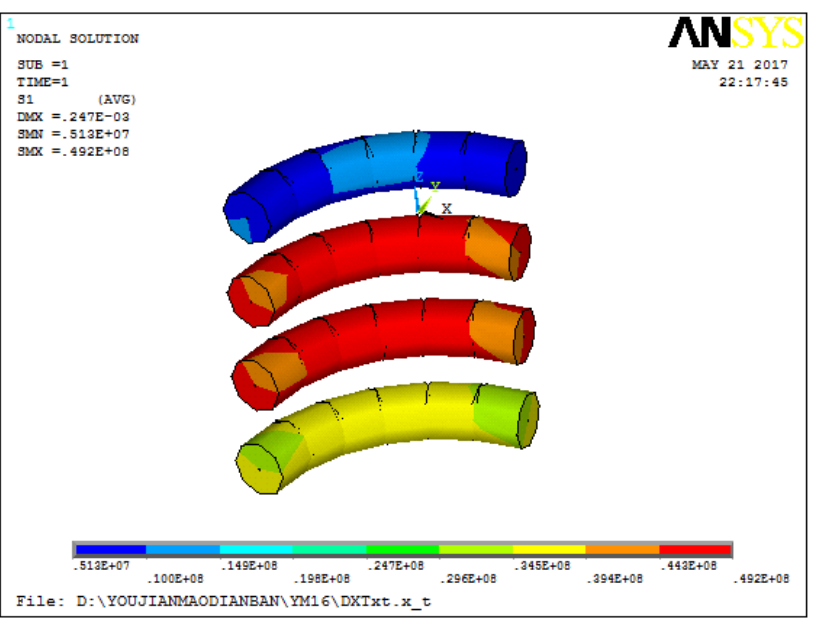

(a)

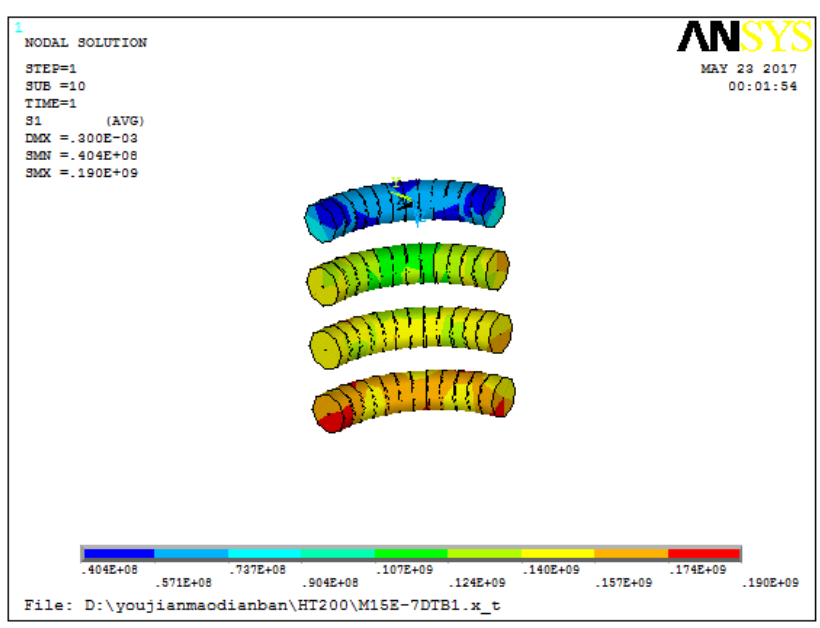

(b)

Figure 5. Stress cloud of (a) YM15-7D (HJ) spiral tendons and (b) M15E-7D (TB) spiral tendons.

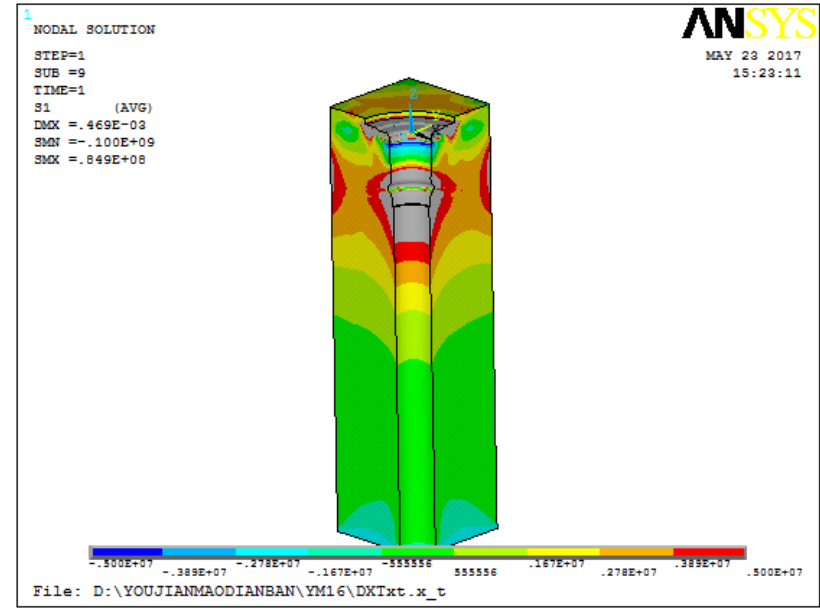

(a)

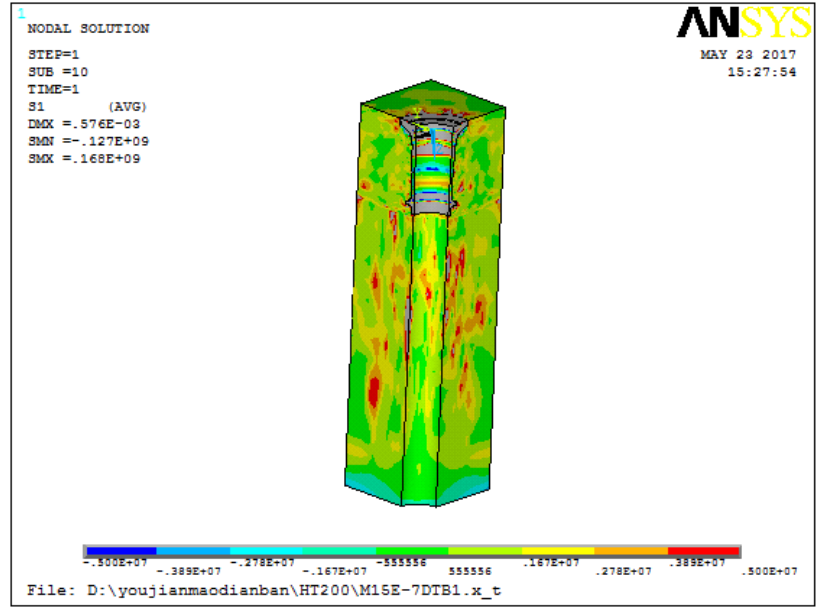

(b)

Figure 6. Concrete stress cloud of (a) YM15-7D (HJ) and (b) M15E-7D (TB). 


\section{Conclusions}

1) This paper designed the quality for new type anchor bearing plate about 3 $\mathrm{kg}$ and cast iron anchor bearing plate $4.74 \mathrm{~kg}$. Therefore, the quality of a new type of anchor bearing plate is reduced by $1.74 \mathrm{~kg}$ compared with the cast iron anchor bearing plate. Currently, with China's rapid development of economy impels the average annual consumption of anchor bearing plate, steel and cost can be greatly saved with marketing this new type anchor bearing plate.

2) Casting anchor bearing plate made of HT200 cast iron that has a strong brittleness, and once the stress exceeds the ultimate strength, it will be brittle damage. The improved new type anchor bearing plate using Q235 steel has a strong elastic-plastic, even if the stress exceeds the ultimate bearing capacity, it will be deformed without breaking and can continue to play a supporting role. According to the numerical simulation analysis of ANSYS finite element, we can see that the actual concrete structure under normal circumstances is relatively safe. The strength of the new type anchor bearing plate is sufficient and the spiral tendons also play a certain role of partial pressure.

\section{References}

[1] JGJ85 (2010) Technical Specification for Application of Anchorage, Grip and Coupler for Prestressing Tendons. China Construction Industry Press, Beijing. (In Chinese)

[2] Qian, M.A., Zhu, W.X., Su, H.T., et al. Optimization Design for Prestressed Bearing Plate Structure. Journal of Sichuan University of Science and Technology, 4, 81-87. (In Chinese)

[3] Zhuang, Z. (2006) Prestressed Structure Anchorage-Contact Mechanics and Engineering Application. Science Press, Beijing. (In Chinese)

[4] Yin, S.Q. (2003) Finite Element Analysis and Experimental Study of Prestressed Anchorage System. South China University of Technology, Guangzhou. (In Chinese)

[5] Lrfd, A. (2012) Bridge Design Specifications.

[6] Gui, Z.G. (2010) Study of Tendon Anchorage Zones of Containment Vessel in Nuclear Power Station and Optimization of Anchor Plate. Guangxi Institute of Technology. (In Chinese) 